\title{
Diagnostik und Therapie von Verletzungen am proximalen Femur und Beckenavulsionsverletzungen bei Kindern sowie der Epiphysiolysis capitis femoris
}

Kai Ziebarth, Nadine Kaiser, Thoralf Liebs

\section{Vorspann}

Obwohl bei Kindern Verletzungen des proximalen Femurs und Avulsionsfrakturen des Beckens sowie die Epiphysiolysis capitis femoris eine niedrige Inzidenz aufweisen, können bei verspäteter Diagnose oder insuffizientem Management langfristig schwerwiegende Einschränkungen bei den betroffenen Kindern auftreten. Dieser Beitrag legt anschaulich dar, worauf bei der Diagnostik und modernen Therapie geachtet werden sollte.

\section{Einleitung}

Grundsätzlich unterscheidet sich die Behandlung von kindlichen Frakturen aufgrund der biologischen Besonderheit des kindlichen Knochens und der offenen Wachstumsfugen von der Frakturbehandlung des Erwachsenen. Durch den deutlich elastischeren Knochen und dem dickeren Periostschlauch kommt es im Falle einer Fraktur selten zu Trümmerfrakturen. Aufgrund der unterschiedlichen Anteile der Wachstumsfugen am Längenwachstum des Knochens kann es bei Kindern zu einer Korrektur von Restfehlstellungen nach einer Fraktur kommen (Remodeling), wenn die entsprechende Wachstumsfuge nahe der Fehlstellung einen großen Anteil am Längenwachstum des Knochens hat und die entsprechende Fehlstellung in der Hauptbewegungsrichtung des angrenzenden Gelenkes liegt. Da die Wachstumsfugen am proximalen Femur bei Kindern mit etwa 13\% nur einen kleinen Anteil am weiteren Längenwachstum beisteuern, erfolgt in diesem Bereich nur eine unzureichende Korrektur einer Restfehlstellung durch das Wachstum [1]. Somit muss im Falle von Frakturen am proximalen Femur eine anatomische Reposition erfolgen, sodass eine konservative Behandlung lediglich bei stabilen und undislozierten Frakturen möglich ist. Das bedeutet, dass der Anteil der Frakturen am proximalen Femur, die operativ versorgt werden müssen, viel höher ist als bspw. am distalen Radius, wo Fehlstellungen nach Frakturen akzeptiert werden können, da hier ein sehr großes Korrekturpotenzial vorliegt.

Eine weitere biologische Besonderheit bei Kindern stellen die Apophysen dar. Im Gegensatz zu den Epiphysenfugen der Röhrenknochen, die ausschließlich für das Längenwachstum Sorge tragen, stellen die Apophysen Muskelansatzpunkte dar und nehmen nicht oder nicht signifikant am Längenwachstum teil. Die Apophysen stellen je nach Alter des Kindes mechanische Schwachstellen dar, sodass die an den jeweiligen Apophysen ansetzenden Muskeln oder Sehnen dort knöchern ausreißen können, da aufgrund der biomechanischen Besonderheiten bei Kindern die Bänder resp. Sehnen mechanisch kräftiger sind als der Knochen selbst. Somit kommt es bei Kindern selten zu Bandläsionen oder Sehnenrissen, sondern eher zu knöchernen Ausrissen (Avulsionen) von Bändern oder Sehnen an den Ansatzpunkten.

Eine ähnliche Ursache wie bei den Apophysenverletzungen wird bei der Epiphysiolysis capitis femoris (ECF) vermutet. Auch hier stellt die Wachstumsfuge eine mechanische Schwachstelle dar und ist für das Abrutschen des Femurkopfes mit verantwortlich.

Sehr häufig klagen Kinder auch über muskuläre Schmerzen, die infolge des steten Längenwachstums des Kindes durch eine relative Verkürzung der Muskulatur hervorgerufen werden können. Hier kommt es typischerweise zu Schmerzen z. B. an der Tuberositias tibiae sowie am Tuber calcanei durch eine Verkürzung der Quadrizeps- oder der Gastrocnemiusmuskulatur. Auch am proximalen Femur und am Beckenkamm kann es durch Muskelverkürzungen der dort ansetzenden Muskeln (M. psoas, M. sartorius, M. rectus femoris) zu Beschwerden kommen. Auch der M. tensor fasciae latae kann im Rahmen des Wachstums verkürzt sein und dann, vor allem bei Mädchen, eine schnappende Hüfte (Coxa saltans) hervorrufen.

In diesem Artikel geht es um die Behandlung der schwerwiegenderen Verletzungen des proximalen Femurs wie Frakturen, Apophysenausrisse oder Femurkopfgleiten (ECF) und weniger um die wachstumsbedingten muskulären Dysbalancen.

\section{Proximale Femurfrakturen}

Verletzungen des proximalen Femurs zählen mit einer Inzidenz von $0,5 \%$ bis $1 \%$ zu den seltenen Verletzungen im Kindesalter. Da es sich häufig um Hochrasanzverletzungen handelt, haben die Kinder nicht selten weitere Verletzungen an Kopf oder Brust-/Bauchorganen und benötigen dementsprechende weitere Untersuchungen. Trotz, oder gerade wegen der Seltenheit der Fraktur ist die Kenntnis der korrekten Versorgung essenziell. Das 
größte Komplikationsrisiko dieser Frakturen liegt in der Entwicklung einer avaskulären Nekrose (AVN) des Femurkopfes. Diese kann das Kind in seiner Bewegungsfreiheit, sei es für sportliche Aktivitäten oder in beruflichen Belangen, ein Leben lang einschränken. Da das Korrekturpotenzial von Fehlstellungen am proximalen Femur sehr begrenzt ist, kommt daher der korrekten anatomischen Reposition mit stabiler Fixation bei diesen Frakturen eine hohe Bedeutung zu.

\section{Entwicklung und Anatomie}

Zum proximalen Femur zählt der proximale Femurschaft, die daran angrenzende metaphysäre Region inkl. der Trochanteren sowie der Schenkelhals und der Femurkopf. Der CCD-Winkel (Centrum-Collum-Diaphysenwinkel) ist altersabhängig und beträgt beim Neugeborenen etwa $160^{\circ}$, beim Schulkind $135^{\circ}$ und beim Adoleszenten und Erwachsenen $125^{\circ}$. Dies ist für die korrekte Reposition der Frakturen zu berücksichtigen.

Ein kritischer Aspekt bei allen Erkrankungen und Verletzungen des proximalen Femurs ist die Blutversorgung in dieser Region. 80\% der Durchblutung der Femurkopfepiphyse verläuft über die A. circumflexa medialis, die dorsal am Trochanter major aufsteigt und am Oberrand des Schenkelhalses von posterolateral in den Femurkopf zieht. Hier ist sie bei Schenkelhalsfrakturen besonders gefährdet. Bei Verletzungen des Gefäßes resultiert eine avaskuläre Nekrose des Femurkopfes [2].

\section{Bildgebung}

Bei Verdacht auf eine Schenkelhalsfraktur erfolgt zunächst ein Röntgen des Beckens a.-p. sowie eine ergänzende axiale Aufnahme des verletzten Hüftgelenkes ( $\triangleright$ Abb. $1 \mathbf{a}$ und b). Eine Aufnahme nach Lauenstein ist nicht zu empfehlen, da dafür das Femur rotiert werden muss und es massive Schmerzen für den Patienten hervorrufen kann und die Fraktur ggf. noch mehr disloziert wird.

Eine weitere Bildgebung, vorzugsweise mittels MRT aufgrund der niedrigeren Strahlenbelastung, ist nur bei einem Verdacht auf eine intraartikuläre Beteiligung, unklaren Befunden im Standardröntgenbild oder Diskrepanz zwischen einem unauffälligen Röntgenbild und schmerzgeplagtem Patienten notwendig. Alternativ kann auch ein CT erfolgen.

Die Klassifikation der Frakturen erfolgt nach Delbet oder der AO-Klassifikation für Kinder [3] ( Tab. 1).

\section{Behandlung}

Die Behandlung der proximalen Femurfraktur beim Kind erfolgt in den überwiegenden Fällen operativ und richtet sich nach der Art der Fraktur. Prinzipiell ist jedoch immer eine anatomische Reposition mit stabiler Fixation anzustreben. Bei der Versorgung sollte immer bedacht werden, dass sich bei den Typ-I-III-Frakturen immer ein ausgeprägter Bluterguss in der Gelenkkapsel des Hüftgelenkes bildet. Durch den so erhöhten intraartikulären Druck kann die Durchblutung des Femurkopfes zusätzlich beeinträchtigt werden. Wir empfehlen, bei Schenkelhalsfrakturen immer eine Arthrotomie mit einer kontrollierten offenen Reposition zum Schutz der Blutversorgung und zur Entlastung des Frakturhämatoms.

\section{Delbet-Typ-I-Frakturen}

Nur unverschobene Brüche beim kleinen Kind (<2 Jahre) können konservativ im Becken-Bein-Gips behandelt werden. Alle anderen Frakturen dieser Gruppe müssen reponiert und fixiert werden. Als Operationsmethoden wer-
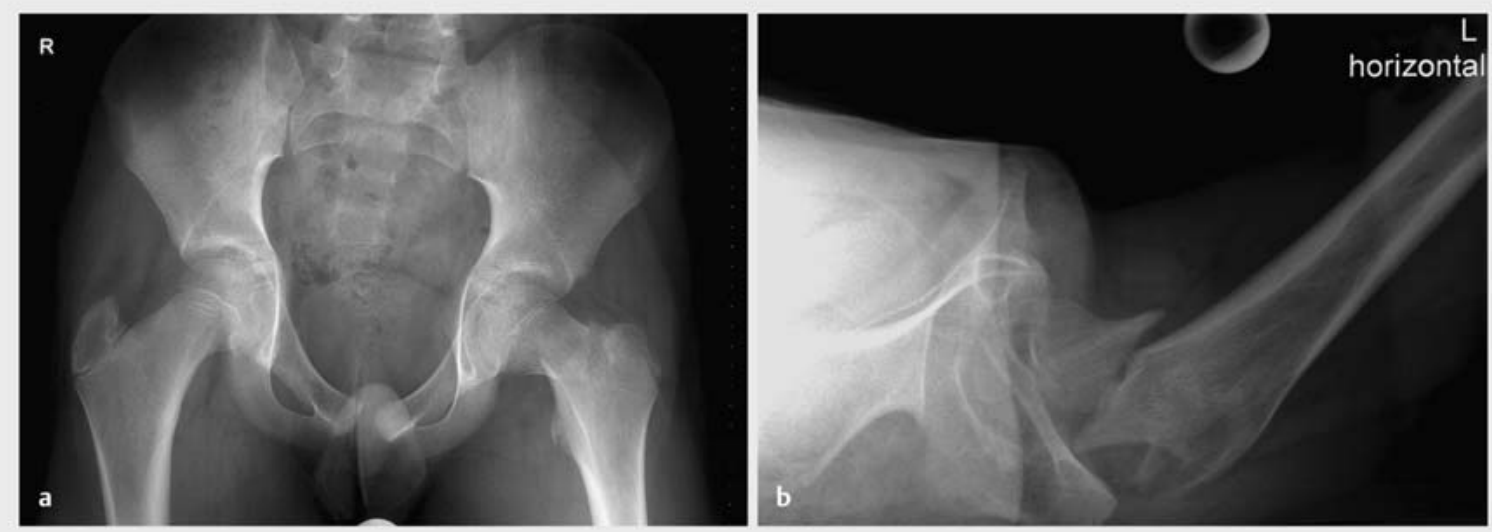

- Abb. 1 a, b 12-jähriger Junge mit dislozierter medialer Schenkelhalsfraktur AO 31-M/3.1I (Delbet Typ II) auf der linken Seite nach Skisturz. 
- Tab. 1 Klassifikation der proximalen Femurfrakturen gemäß AO-Klassifikation für Kinder und Delbet-Klassifikation inkl. ihrer Häufigkeit und Rate der avaskulären Nekrose des Femurkopfes (AVN).

\begin{tabular}{|c|c|c|c|c|}
\hline AO-Klassifikation & $\begin{array}{l}\text { Delbet- } \\
\text { Klassifikation }\end{array}$ & Typ & $\begin{array}{l}\text { AVN- } \\
\text { Rate }\end{array}$ & $\begin{array}{l}\text { Häufig- } \\
\text { keit }\end{array}$ \\
\hline $31-E / 1.1$ & \multirow[t]{2}{*}{ IA } & transepiphysäre Fraktur ohne Dislokation & \multirow[t]{3}{*}{$40 \%$} & \multirow[t]{3}{*}{$8 \%$} \\
\hline $31-E / 2.1$ & & transepiphysäre Fraktur mit metaphysärem Keil & & \\
\hline $31-E / 2.1$ & IB & transepiphysäre Fraktur (jede) mit Dislokation & & \\
\hline $31-\mathrm{M} / 2.11$ & \multirow[t]{2}{*}{ II } & inkomplette mediale Schenkelhalsfraktur & \multirow[t]{2}{*}{$27 \%$} & \multirow[t]{2}{*}{$45-50 \%$} \\
\hline $31-M / 3.11$ & & mediale Schenkelhalsfraktur & & \\
\hline $31-\mathrm{M} / 2.1 \mathrm{II}$ & \multirow[t]{3}{*}{ III } & inkomplette basizervikale/laterale Schenkelhalsfraktur & \multirow[t]{3}{*}{$20 \%$} & \multirow[t]{3}{*}{$34 \%$} \\
\hline $31-\mathrm{M} / 3.1 \mathrm{II}$ & & einfache laterale Schenkelhalsfraktur & & \\
\hline $31-\mathrm{M} / 3.2 \mathrm{II}$ & & mehrfragmentäre laterale Schenkelhalsfraktur & & \\
\hline $31-\mathrm{M} / 2.1 \mathrm{III}$ & \multirow[t]{3}{*}{ IV } & inkomplette pertrochantäre Fraktur & \multirow[t]{3}{*}{$5 \%$} & \multirow[t]{3}{*}{$12 \%$} \\
\hline $31-\mathrm{M} / 3.1 \mathrm{III}$ & & einfache pertrochantäre Fraktur & & \\
\hline $31-\mathrm{M} / 3.2 \mathrm{III}$ & & mehrfragmentäre pertrochantäre Fraktur & & \\
\hline $31-\mathrm{M} / 7$ & $\%$ & Avulsionsfrakturen des proximalen Femurs & & \\
\hline
\end{tabular}

den in der Literatur die geschlossene Reposition mit anschließender Fixation mittels Kirschner-Drähten oder kanülierten Schrauben sowie die offene Reposition und Fixation mittels Schrauben oder der pädiatrischen Hüftplatte empfohlen [1].

\section{Delbet-Typ-II- und -III-Frakturen}

Diese klassischen Schenkelhalsfrakturen benötigen immer eine operative Stabilisierung. Als Operationsmethoden werden die offene oder geschlossene Reposition mit Schraubenosteosynthese oder Kirschner-Drähten sowie die offene Reposition mit Stabilisierung durch eine pädiatrische Hüftplatte oder Schraubenosteosynthese genannt [1]. Wir bevorzugen die offene Reposition und Stabilisierung mit pädiatrischer Hüftplatte ( $\bullet$ Abb. $\mathbf{2 a}$ und b). Bei Typ-II-Frakturen sollten die Schrauben zur besseren Stabilität über die Epiphysenfuge eingebracht werden.

\section{Delbet-Typ-IV-Frakturen}

Diese pertrochantären Frakturen können beim kleinen Kind (<2 Jahre) konservativ im Becken-Bein-Gips oder bei dislozierten Frakturen in der Overhead-Extension behandelt werden ( $\boldsymbol{A}$ Abb. $\mathbf{3} \mathbf{a}-\mathbf{d})$. Ältere Kinder sowie dislozierte Frakturen benötigen eine operative Reposition mit anschließender Stabilisation mittels pädiatrischer Hüftplatte oder Schraubenosteosynthese [1]. Subtrochantäre Frakturen können, bei entsprechender Kenntnis und Übung in der Technik, auch mittels elastisch stabiler intramedullärer Nagelung (ESIN) versorgt werden [4].

\section{Komplikationen}

Die häufigste Komplikation dieser Frakturen ( $\bullet$ Tab. 1$)$ ist die AVN des Femurkopfes. Die Ursache ist meist eine Ver-
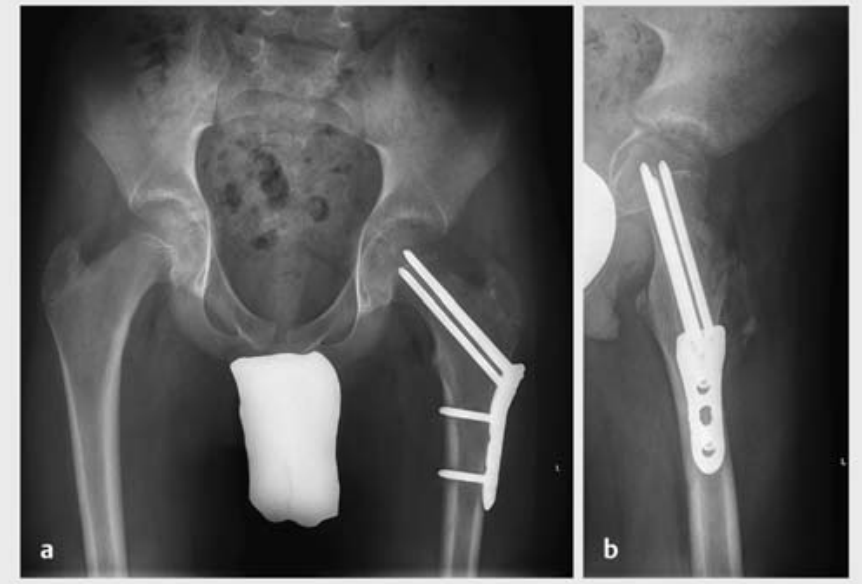

Abb. 2 a, b Versorgung einer medialen Schenkelhalsfraktur AO 31-M/ 3.11 mit einer LCP pädiatrischen Hüftplatte $5.0130^{\circ}$.

letzung der retinakulären Gefäße durch den Unfall. Aber auch wiederholte Repositionsmanöver beim Versuch der geschlossenen Reposition, unvorsichtige Bewegungen der verletzten Extremität sowie ein intrakapsuläres Hämatom oder iatrogene Gefäßverletzungen bei operativen Eingriffen können ursächlich sein. Gemäß der aktuellen Literatur steigt das Risiko einer AVN mit dem Ausmaß der Dislokation, dem Alter des Kindes und der Zeit bis zur Stabilisierung der Verletzung ( $>24 \mathrm{~h}$ ) an, hingegen sinkt die Rate der AVN, je stabiler die definitive Versorgung ist und je besser die Fraktur reponiert wurde [5]. Des Weiteren hat sich gezeigt, dass die rasche Versor- 

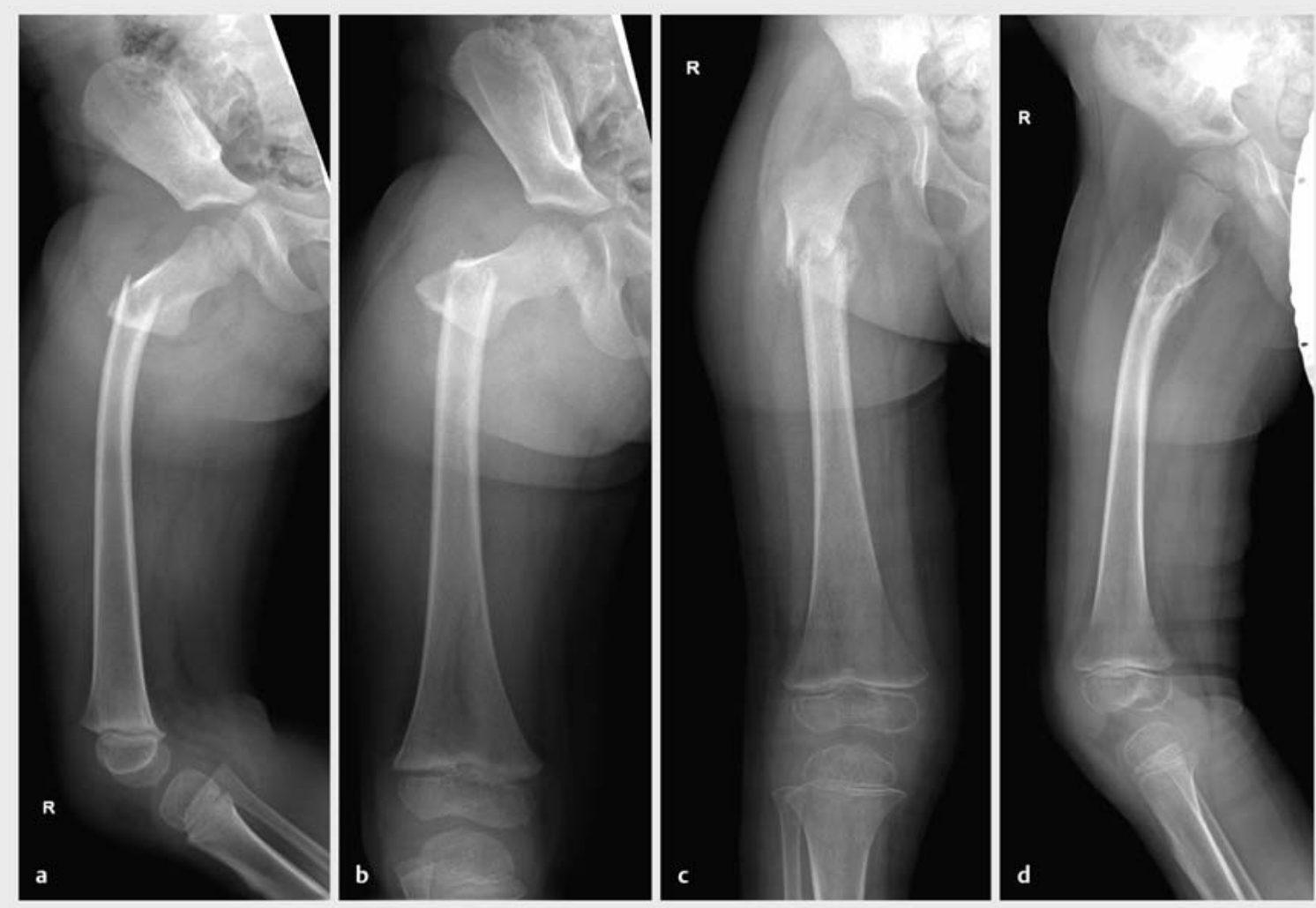

- Abb. 3 a, b Subtrochantäre Femurfraktur bei einem 3²/2-jährigen Knaben. c, d Ausheilungsergebnis der proximalen Femurfraktur ( $\triangleright$ Abb. $\mathbf{3}$ a und $\mathbf{b}$ ) nach konservativer Behandlung in der Overhead-Extension während 4 Wochen.

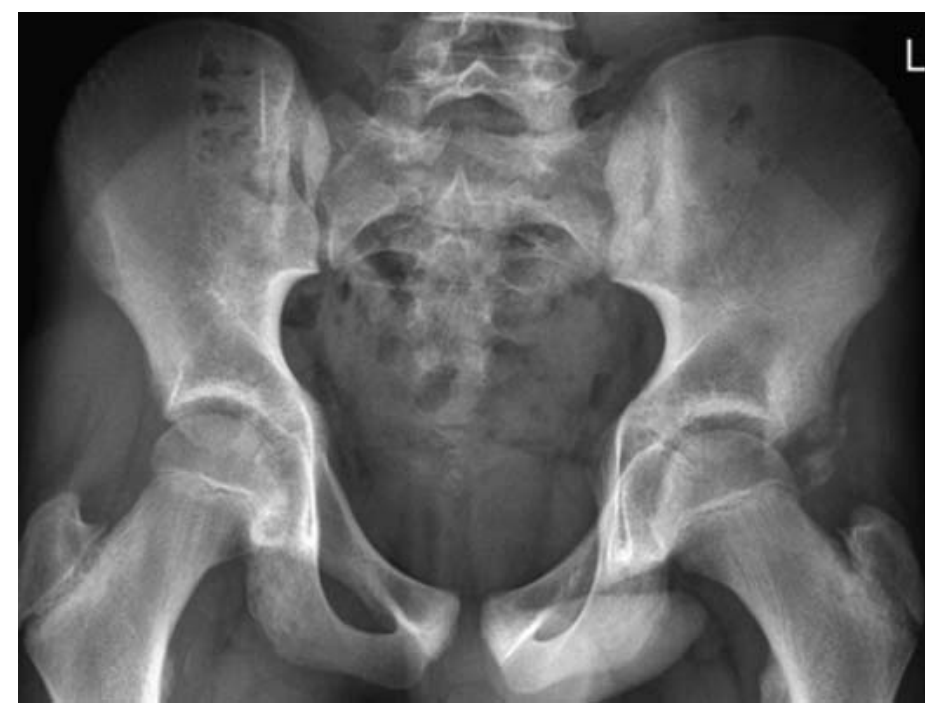

- Abb. 4 Avulsionsfraktur der Spina iliaca anterior inferior bei einem 15jährigen Fußballspieler. gung durch einen hüfterfahrenen Operateur ein wichtiger prognostischer Faktor ist.

Weitere Komplikationen sind:

- Das Entstehen einer Coxa vara durch Abrutschen des Femurkopfes (18\%) bei nicht ausreichend stabilisierten Brüchen [6]. Bei Kindern über 8 Jahren werden aufgrund des geringen Korrekturpotenzials valgisierende Korrekturosteotomien bei Coxa vara empfohlen.

- Vorzeitiger Fugenverschluss (13-15\%) [6]: Dieser ist jedoch aufgrund des geringen Anteils am Längenwachstum nur bei zum Unfallzeitpunkt jungen Kindern relevant.

- Nonunion (11\%) [5]: Die Pseudarthrose ist bei Kindern eine seltene Komplikation und i.d.R. Zeichen einer ungenügend stabilisierten Fraktur. Wenn nach 3-6 Monaten noch immer Schmerzen bestehen und im CT eine nicht geheilte Fraktur bestätigt wird, besteht die Indikation zur Revision. 


\section{Apophysenfugenverletzungen am proximalen Femur und Becken}

Verletzungen der Apophysenfugen am Becken und proximalen Femur treten typischerweise bei sportlichen Belastungen auf, die mit plötzlichen starken Muskelkontraktionen einhergehen, wie z. B. beim Springen, Laufen oder beim Fußballspielen. Dabei kommt es aufgrund der starken Zugbelastung zu einem Versagen der Apophysenfuge, sodass das sekundäre Ossifikationszentrum an der Apophysenfuge aus seinem Bett gelöst wird.

Leider liegen in der Literatur nur widersprüchliche Angaben zur Inzidenz vor. Diese ist auch abhängig von der Patientenselektion, insbesondere auch vom Alter der Patienten und von der Lokalisation der Verletzung. Die ersten sekundären Ossifikationszentren an den unteren Extremitäten treten bereits ab dem 4. Lebensjahr (Trochanter major) auf und fusionieren i.d. R. bis zum 17. Lebensjahr. Allerdings findet die Fusion bei der Spina iliaca anterior superior und der Tuberositas ischii erst zum 25. Lebensjahr statt.

In der Literatur wird beschrieben, dass Beckenavulsionsfrakturen dieser Regionen überwiegend (zu 76\%) bei Jungen vorkommen [7] und dass - je nach Autor - die Spina iliaca anterior inferior, die Tuberositas ischii oder die Spina iliaca anterior superior am häufigsten betroffen sind, während Avulsionsverletzungen der Trochanteren und des Beckenkamms deutlich seltener auftreten [7].

Der Unfallmechanismus ist häufig hinweisend auf die jeweilige Verletzung, wobei später im Artikel auf die jeweiligen Besonderheiten, sowohl hinsichtlich der Therapie als auch der Komplikationsmöglichkeiten, eingegangen wird.

Neben der körperlichen Untersuchung ist i.d. R. eine nativradiologische Diagnostik ausreichend. Nur in seltenen Fällen ist eine weitere Diagnostik, wie z. B. ein MRT bei V.a. Avulsionsverletzungen bei bislang noch knorpelig ausgebildeten Ossifkationszentren oder bei V.a. Mitverletzung des Labrum acetabulare, erforderlich. Die Rolle der Sonografie in der Primärdiagnostik wird aktuell noch kontrovers angesehen.

Eine Klassifikation der Avulsionsverletzungen wurde von McKinney [8] vorgeschlagen. Dabei ist eine Typ-I-Verletzung nicht disloziert, eine Typ-II-Verletzung um maximal $2 \mathrm{~cm}$, eine Typ-III-Verletzung um mehr als $2 \mathrm{~cm}$ disloziert und eine Typ-IV-Verletzung weist eine symptomatische Pseudarthrose bzw. eine schmerzhafte heterotope Ossifikation auf.

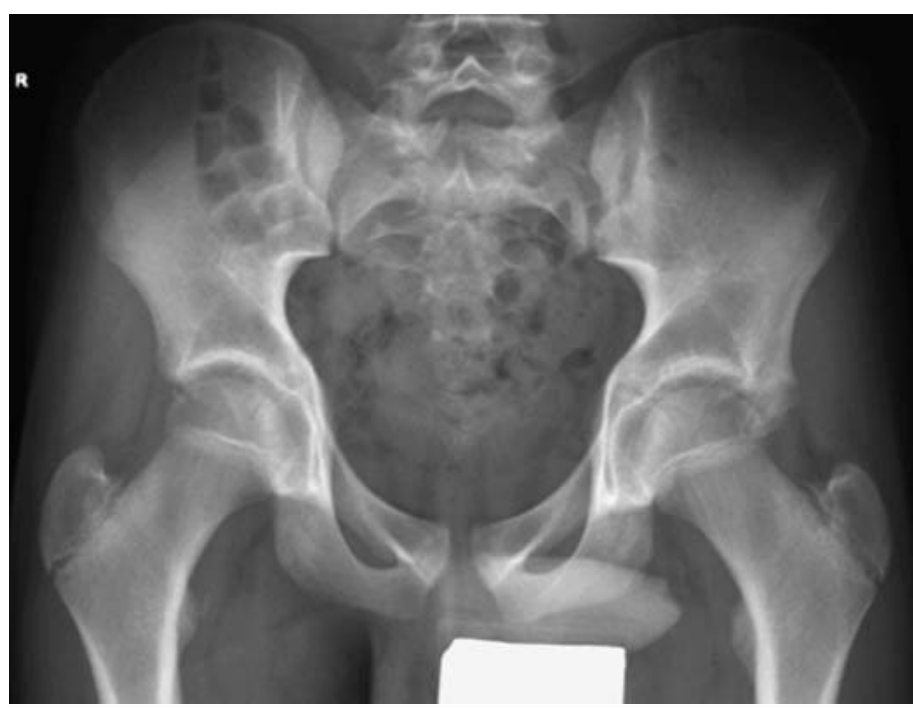

- Abb. 5 Sieben Monate nach dem Unfall besteht eine vollständige knöcherne Konsolidierung, der Junge gibt keine Schmerzen an und die Beweglichkeit ist frei.

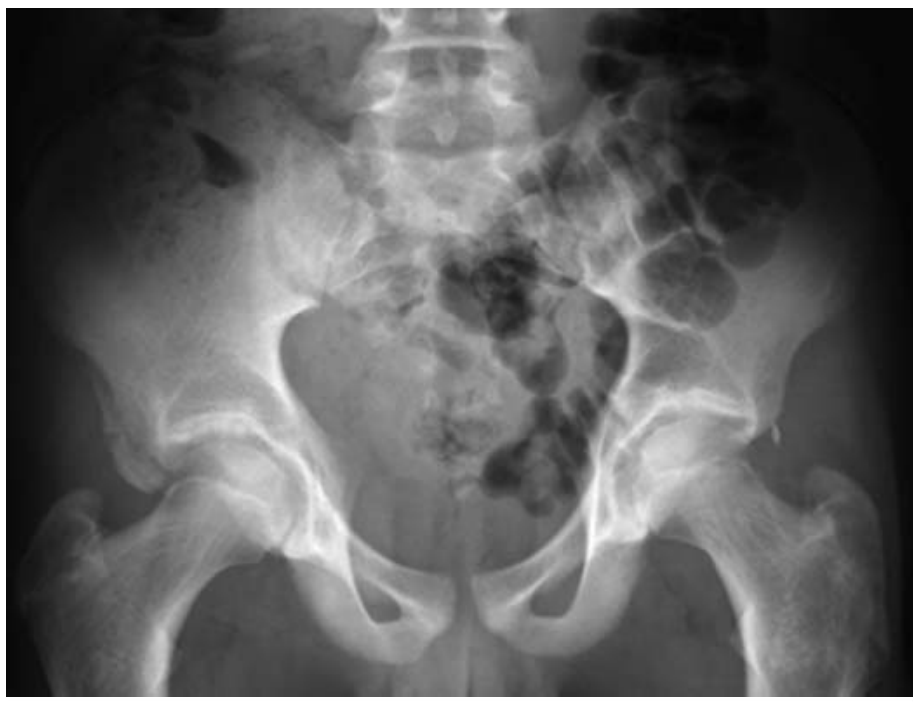

- Abb. 6 Extraartikuläres Impingement nach einer Fraktur der Spina iliaca anterior inferior rechts bei einem 14-jährigen Eishockeyspieler.

\section{Spina iliaca anterior inferior}

Der $M$. rectus femoris entspringt mit seinem geraden Kopf an der Spina iliaca anterior inferior. So ist es auch zu erklären, dass Verletzungen dort i.d.R. durch kraftvolle Knieextension und Hüftflexion zustande kommen ( Abb. 4).

Die Therapie dieser Verletzung ist initial üblicherweise konservativ, bestehend aus Entlastung an 2 Unterarmgehstützen. Nach dem bereits 1985 veröffentlichten 5Stufen-Protokoll von Metzmaker und Pappas [9] wird 


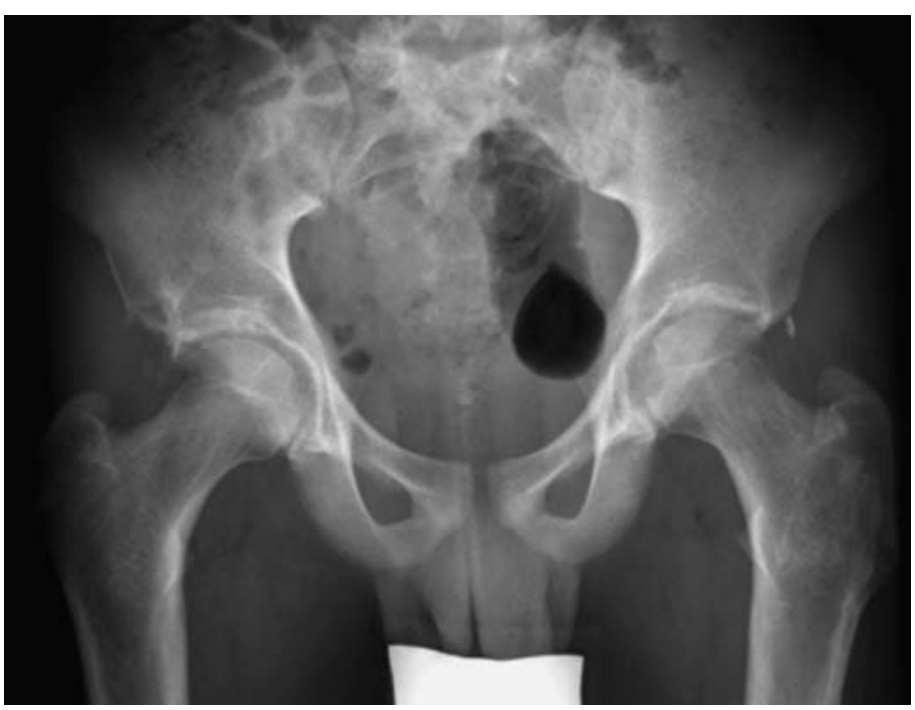

Abb. 7 Zustand nach Resektion des Fragments rechts.

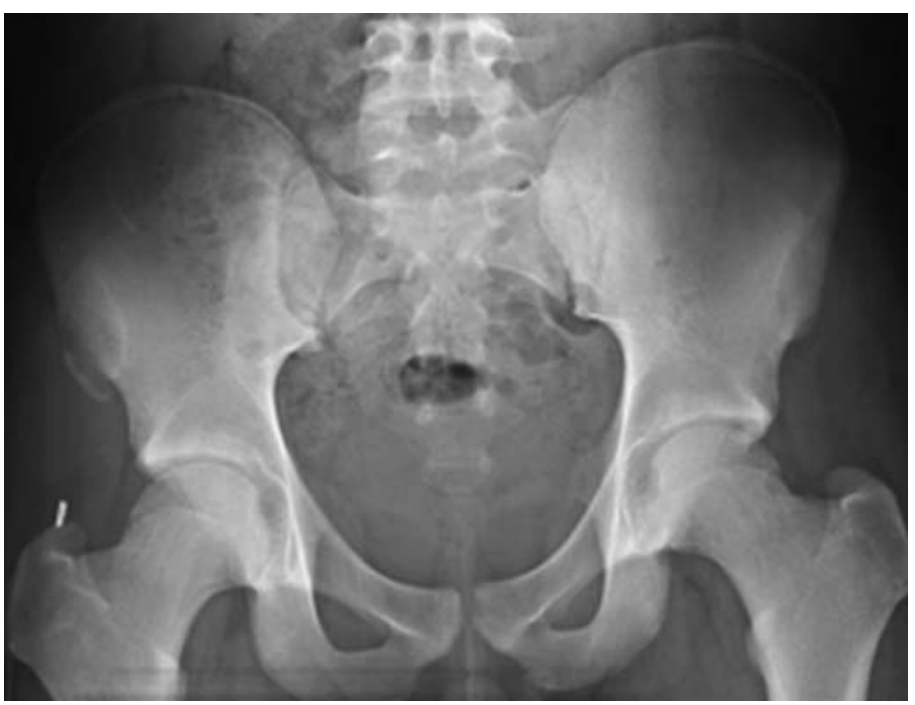

- Abb. 8 Avulsionsverletzung der Spina iliaca anterior superior rechts, die konservativ therapiert worden ist.

empfohlen, in der 1. Woche eine vollständige Entlastung einzuhalten, in der 2. bis 4 . Woche können passive Bewegungen durchgeführt werden und eine Teilbelastung ist gestattet. Ab Woche 4 bis 6 können aktive Bewegungen durchgeführt werden, inkl. isokinetischer Übungen. Ab der 6. bis 9. Woche sind Übungen gegen Widerstand, und ab der 9 . Woche sind die gewohnten Aktivitäten wieder gestattet.
In der Mehrzahl der Fälle kann so eine vollständige Rekonvaleszenz binnen 3 Monaten erzielt werden [9] ( $\triangleright$ Abb. 5).

Allerdings wurde in der Literatur beschrieben, dass Frakturen der Spina iliaca anterior inferior rund 4-mal häufiger als andere Avulsionsfrakturen des Beckens zu einer Beschwerdepersistenz nach 3 Monaten führen können und dass eine Dislokation von mehr als $2 \mathrm{~cm}$ ein 26-fach erhöhtes Risiko einer Pseudarthrose aufweisen würde [7]. Des Weiteren besteht auch die Möglichkeit eines extraartikulären Impingements, welches bei Beschwerden eine Resektion des Fragments erforderlich machen kann ( $\bullet$ Abb. 6 und 7).

Ebenso kann im Rahmen dieser Verletzung auch der anterosuperiore Anteil des Labrums verletzt werden, sodass sich ein femoroazetabuläres Impingement mit einer sekundären Koxarthrose entwickeln kann.

\section{Spina iliaca anterior superior}

An der Spina iliaca anterior superior entspringt der M. sartorius und der M. tensor fascia lata. Verletzungen in diesem Bereich sind beim Rennen, Springen sowie beim Baseball beschrieben worden ( $\bullet$ Abb. 8).

Auch hier wird üblicherweise eine konservative Therapie empfohlen, bestehend aus nicht steroidalen Antirheumatika (NSAR) und Entlastung des betroffenen Beines für 68 Wochen. Eine chirurgische Refixierung wird nur empfohlen, wenn eine Dislokation von mehr als $2 \mathrm{~cm}$ eingetreten ist. Die verspätete Therapie dieser Verletzungen kann zu einer Kompression des N. cutaneus femoris lateralis mit einer Meralgia paraesthetica führen.

\section{Tuberositas ischii}

Die Tuberositas ischii ist der Ursprung der ischiokrualen Muskulatur („hamstrings“). Dabei handelt es sich um den langen Kopf des M. biceps, um den M. semitendinosus und den M. semimembranosus. Avulsionsverletzungen dieser Region können bei forcierter Hüftbeugung und gleichzeitiger Kniegelenksextension auftreten, wie z. B. beim Rennen und insbesondere beim Fußballspielen ( Abb.9). Diese Verletzung wird häufig initial verkannt bzw. die Patienten stellen sich erst verzögert beim Arzt vor [10].

Wenn zwischenzeitlich weiterhin sportliche Aktivitäten fortgesetzt werden, besteht eine erhöhte Gefahr der Entwicklung einer symptomatischen Pseudarthrose. Dabei ist einerseits das „Hamstring-Syndrom“ zu nennen, wobei die Patienten nicht längere Zeit sitzen können, da Anteile des N. ischiadicus durch die Pseudarthrose oder vernarbte Fasern der Hamstrings eingeengt sein können [10]. Anderseits besteht auch die Gefahr eines ischiofemoralen Impingements, wobei die Tuberositas ischii in Fehlstellung anheilt und zu einem Impingement des N. ischia- 
dicus zwischen diesem Fragment und dem Trochanter minor, insbesondere bei Außenrotation, führt.

Die Behandlung dieser Frakturen erfolgt üblicherweise konservativ unter Stockmobilisation, insbesondere bei einer Dislokation von weniger als $15 \mathrm{~mm}$. Ein Autor beschrieb 13 Patienten, bei denen bei 5 Patienten mit einer Dislokation von mehr als $15 \mathrm{~mm}$ eine operative Refixierung durchgeführt wurde, welche alle sehr gute Ergebnisse aufgewiesen hätten [11].

Hinsichtlich der Therapieverfahren ist eine Vielzahl von Osteosynthesearten beschrieben worden, von der Osteosutur über Schrauben bis zur Plattenosteosynthese. Nach der Osteosynthese wird üblicherweise eine recht lange Rehabilitationsphase empfohlen, wobei für 6 Wochen keine Belastung des betroffenen Beines durchgeführt werden darf und auch empfohlen wird, nicht auf der betroffenen Seite zu sitzen.

\section{Trochanter minor}

Seltene Verletzung. Am Trochanter minor setzt der M. iliopsoas an. Avulsionsfrakturen des Trochanter minor sind daher i.d. R. die Folge von forcierten Beugebelastungen im Hüftgelenk ( $\triangleright$ Abb. 10). Wir sehen diese Frakturen bei Fußballspielern. Hier ist die initiale Therapie konservativ.

\section{Trochanter major}

Die Mm. gluteus medius und minimus als Hüftgelenksabduktoren und ein Teil der „kleinen Außenrotatoren“ setzen am Trochanter major an. In der Literatur wurde beschrieben, dass diese sehr seltene Verletzung typischerweise bei flektiertem und außenrotiertem Hüftgelenk bei adipösen Patienten auftritt. Bei Dislokation des Trochantor major ist eine operative Versorgung indiziert, wie beispielhaft in den folgenden Abbildungen $(\triangleright$ Abb. 11 und 12) dargestellt ist.

\section{Epiphysiolysis capitis femoris (ECF)}

\section{Pathologie}

Die Erstbeschreibung der Erkrankung erfolgte durch Ambroise Paré 1572. Der Femurkopf (Epiphyse) gleitet über den Schenkelhals (Metaphyse) ab. Hierbei verbleibt der Femurkopf jedoch im Azetabulum während der Schenkelhals relativ gesehen zum Femurkopf nach ventral disloziert. Selten kann der Femurkopf auch in Bezug zum Schenkelhals nach lateral abrutschen (Valgus Slip). Die genaue Ursache der ECF ist nicht geklärt. Sicher ist, dass die Wachstumsfuge (Epiphysenfuge) bei der Erkrankung mechanisch geschwächt ist und daher das Abrutschen des Femurkopfes erst ermöglicht wird. Es werden sowohl hormonelle (z. B. Hypothyreose) [12] als auch biomechanische Faktoren (azetabulare Retroversion, Adipositas) [13] für die mechanische Schwächung der Wachstums-

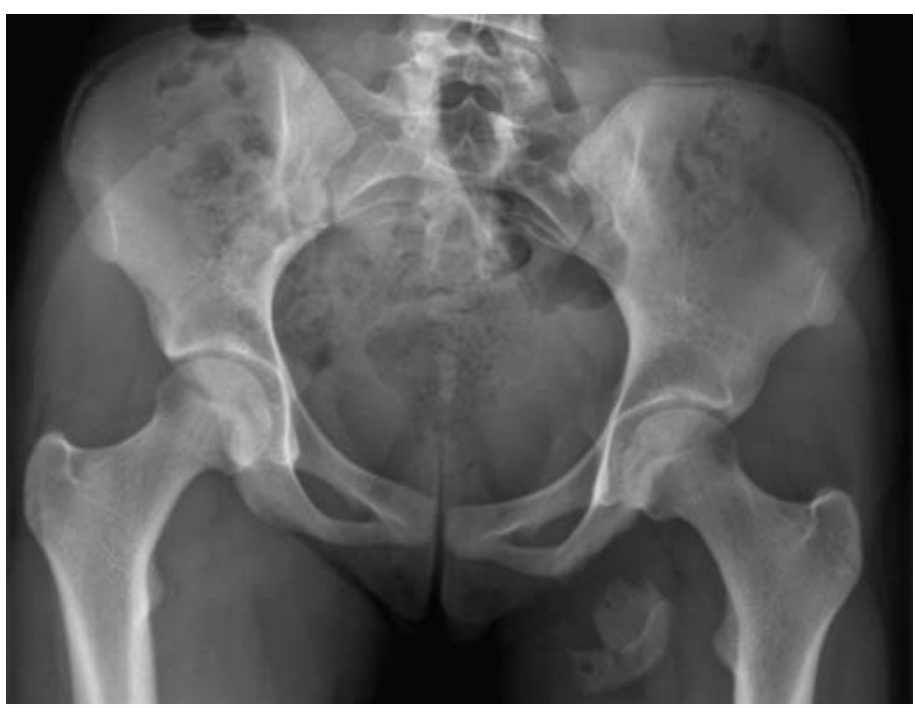

Abb.9 Avulsionsverletzung der Tuberositas ischii links.

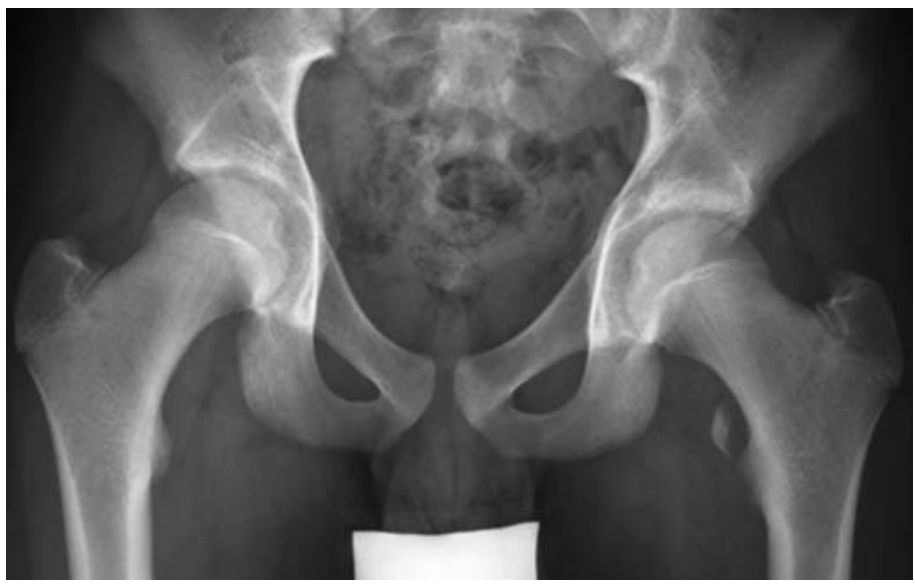

- Abb. 10 Avulsionsfraktur des Trochanter minor links bei einem 15-jährigen Jungen nach Snowboard-Unfall.

fuge und das Abrutschen des Femurkopfes verantwortlich gemacht. Jedoch konnte bisher keine These wissenschaftlich eindeutig bewiesen werden. In seltenen Fällen resultiert eine ECF auch aus einem Trauma. Hierbei kann es einerseits durch die Schwere des Traumas zu einer echten Fraktur mit Epiphysenlösung (Salter-Harris-1-Fraktur), andererseits aber bei bereits bestehender asymptomatischer ECF mit geschwächter Wachstumsfuge zu einem akuten Abrutschen des Femurkopfes kommen. In letzteren Fällen zeigt sich bei der Operation, dass am Schenkelhals bereits Kallusbildung vorliegt, was beweisend für ein bevorstehendes chronisches Geschehen ist und nicht ausschliesslich für eine akute traumatische Ursache spricht. Entscheidend für die Prognose der Erkrankung ist die Stabilität der Wachstumsfuge, d. h. die Ver- 


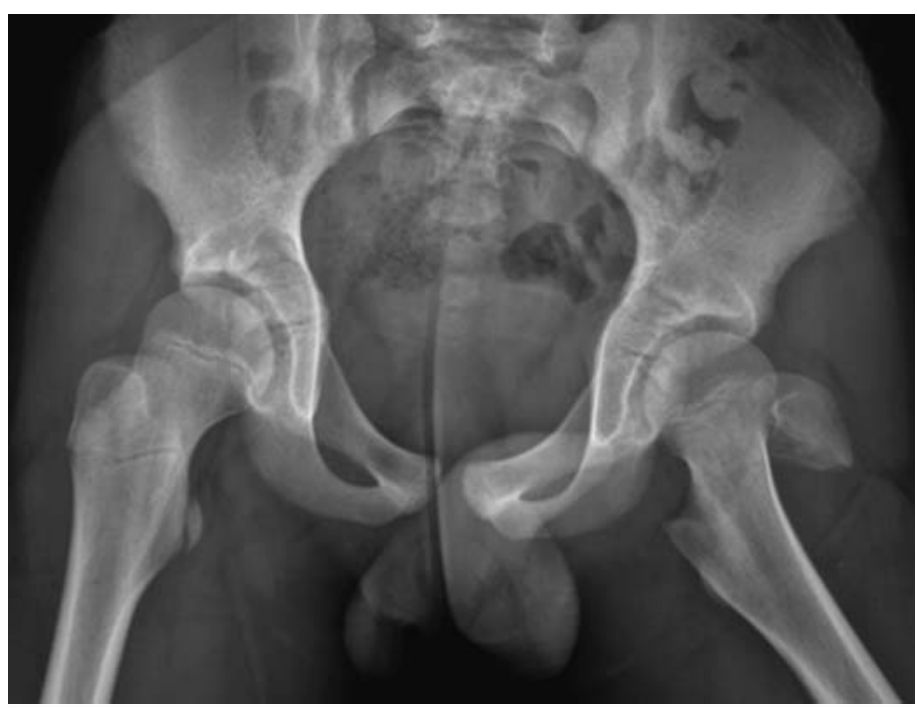

- Abb.11 Avulsionsverletzung des Trochanter major links bei einem 13jährigen Jungen mit einem Hyperflexions- und Außenrotationstrauma beim Skifahren.

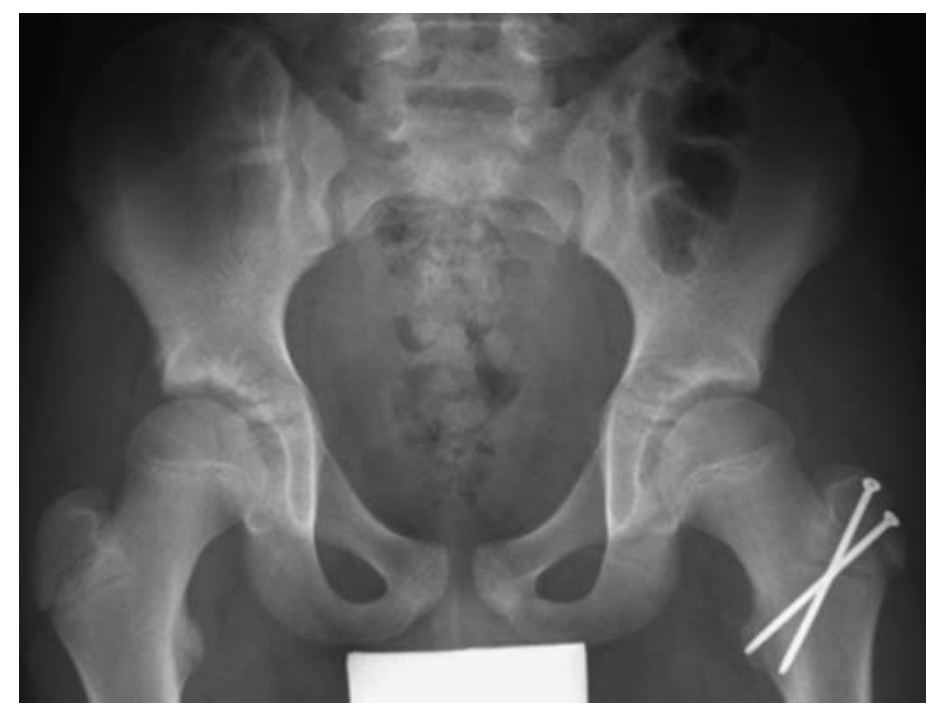

- Abb. 12 Zustand nach offener Reposition und Schraubenosteosynthese des Trochanter major links.

bindung zwischen Schenkelhals und Femurkopf. Ist bei einer instabilen ECF die Verbindung zwischen Femurkopf und Schenkelhals gar nicht mehr oder nicht mehr suffizient vorhanden, droht die weitere Dislokation des Femurkopfes mit Gefährdung der femurkopfversorgenden Blutgefäße. Bei einer stabilen ECF ist eine mechanische Beeinträchtigung des Hüftgelenkes durch die Fehlstellung gegeben, aber ein weiteres akutes Abrutschen mit Gefäßschaden initial nicht zu befürchten. Trotzdem kann auch eine zunächst stabile ECF in eine instabile ECF konvertieren und umgekehrt. Leider stehen bisher keine suf- fizienten klinischen Parameter zur Verfügung, um die Stabilität des Femurkopfes auf dem Schenkelhals klinisch zu beurteilen, sodass jeder Patient mit einer ECF bis zur Stabilisation des Femurkopfes sehr vorsichtig behandelt werden sollte.

\section{Inzidenz}

Die Inzidenz der ECF wird mit etwa 1-10/100000 Fälle in den USA angegeben [14,15]. Diese Zahlen scheinen in etwa mit den Zahlen in Europa übereinzustimmen. In einer Studie von Witbreuk wird die Inzidenz der ECF in den Niederlanden mit 11,6/100000 angegeben [16]. Die Erkrankung tritt gemäß Literatur etwas häufiger bei Jungen als bei Mädchen auf. Das durchschnittliche Alter bei Auftreten der ECF beträgt bei Jungen 13-15 und bei Mädchen 11-13 Jahre (Pubertät), was als Hinweis für eine hormonelle Mitbeteiligung an der Erkrankung angesehen wird. Je nach Literatur kann die Erkrankung initial beidseits auftreten oder kann im Verlauf der nächsten Jahre auch auf der Gegenseite auftreten [17,18]. Daher wird von vielen Autoren, sowie auch von uns die prophylaktische Fixierung der Gegenseite empfohlen.

\section{Klinik}

Während in der englischsprachigen Literatur die Patienten mit ECF meist als übergewichtig beschrieben werden, trifft dies für den mitteleuropäischen Patienten nicht regelhaft zu. In unserem Patientenkollektiv befinden sich sowohl adipöse Patienten mit altersentsprechend zu hohem BMI als auch sportlich schlanke Patienten. Leitsymptome bei der Erstvorstellung beim Kinderarzt sind Knieschmerzen und/oder ein hinkendes, aussenrotierendes Gangbild, meist ohne vorheriges Trauma. Bei der körperlichen Untersuchung ist die eingeschränkte Innenrotation und ein spontanes Außenrotieren des erkrankten Beines bei Hüftbeugung (Drehmann-Zeichen) pathognomonisch. Trotz dieser typischen klinischen Befunde kommt es leider sehr oft vor, dass die Kinder ohne weitere Abklärungen z.B. mit der Diagnose Wachstumsschmerz zunächst konservativ behandelt werden. Somit wird die Diagnosestellung meist über Wochen bis Monate verzögert, was zu einer Zunahme der Fehlstellung mit konsekutiver Schädigung des Hüftgelenkes führen kann. Auch die beweisende Bildgebung wird aus Strahlenschutzgründen von vielen Ärzten zunächst nicht mit in die Abklärung einbezogen oder es wird lediglich nur eine a.-p. Aufnahme des Hüftgelenkes durchgeführt. Dies kann irreführend sein, da in frühen Stadien der Erkrankung die Fehlstellung aufgrund der dorsalen Dislokationsrichtung des Femurkopfes nur in der seitlichen Ebene sichtbar ist ( $\triangleright$ Abb. 13 b).

\section{Bildgebung}

Zur Diagnosesicherung sind eine Beckenübersichtsaufnahme sowie eine zweite Ebene (Lauenstein-Aufnahme oder axiale Aufnahme) notwendig. Um ggf. mehr Informationen über den Gelenkschaden oder die Femurkopf- 


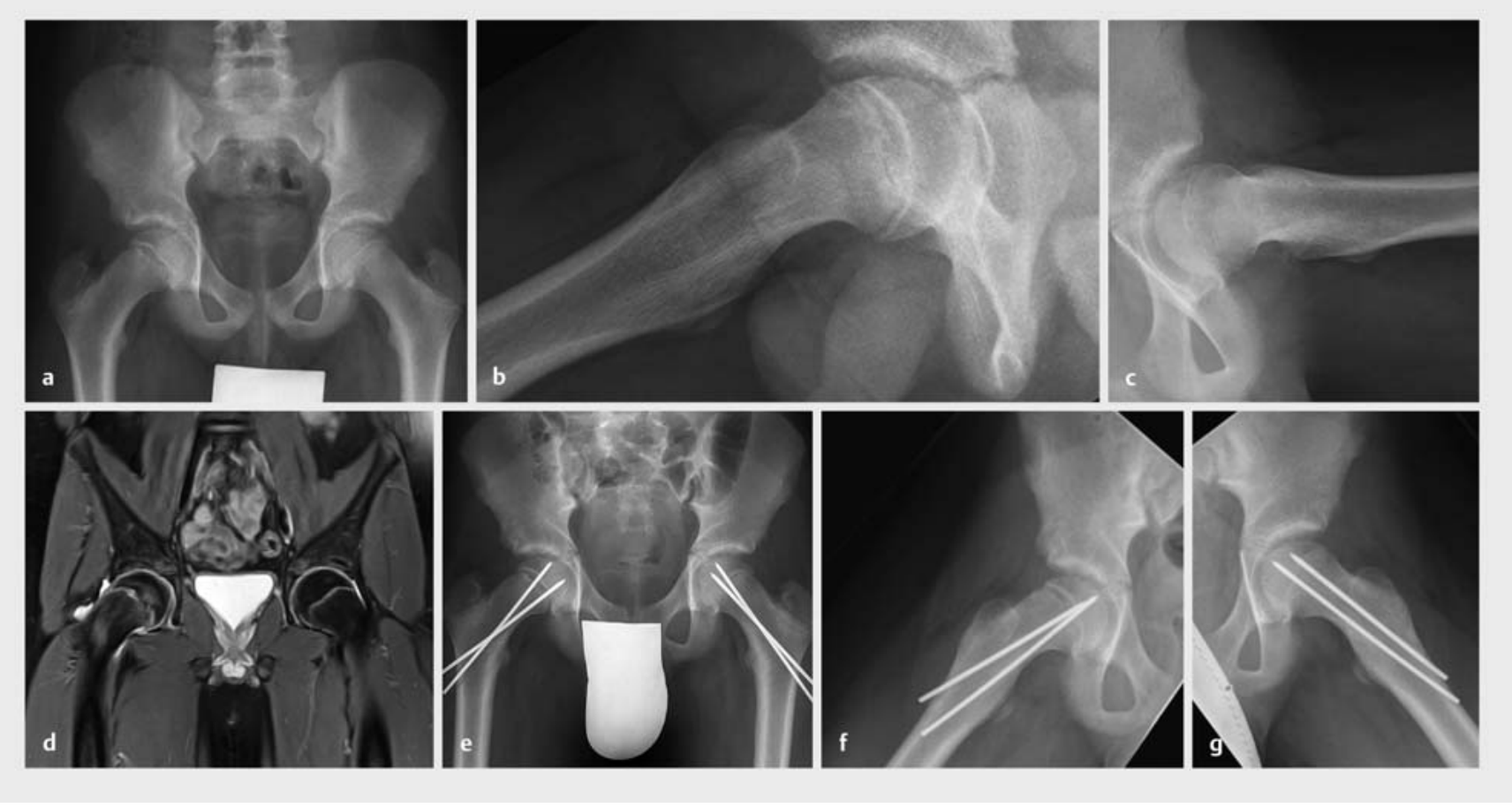

- Abb. 13 Knabe 13 Jahre, ECF rechts: a-c präoperative Aufnahme a.-p. und Lauenstein. Die Auflockerung der Wachstumsfuge der rechten Hüfte ist bereits radiologisch sichtbar. $\mathbf{d}$ MRT: Gelenkerguss rechts (Flüssigkeit weiß), aufgelockerte Wachstumsfuge rechts. e-g Postoperative Aufnahmen a.-p. und Lauenstein bds. nach In-situ-Pinning bds. mit 3,0 mm Vollgewinde-Kirschner-Drähten.

durchblutung zu bekommen, kann eine MRT (Magnetresonanztomographie) zur Behandlungsplanung hilfreich sein.

Die ebenfalls in der Literatur zur Abklärung beschriebene $\mathrm{CT}$ (Computertomographie) ist zur exakten Bestimmung des Abrutschwinkels hilfreich, jedoch aufgrund der hohen Strahlenbelastung für die Kinder eher ungeeignet.

\section{Klassifikation}

Die ECF wird historisch bez. Symptomdauer in akut (Symptome < 3 Wochen), akut auf chronisch (chronische Hüftschmerzen mit Exazerbation innerhalb der letzten 3 Wochen) oder chronisch (chronische Hüftschmerzen $>3$ Wochen) eingeteilt [19]. Eine 1993 publizierte Klassifikation [20] von Loder et al. teilt die ECF nach der mechanischen Stabilität der Epiphysenfuge in stabil oder instabil ein. Es sei erwähnt, dass diese sehr häufig verwendete Klassifikation auf retrospektiven Daten beruht und die mechanische Stabilität lediglich anhand von Röntgenbildern beurteilt wurde. Eine eigene Studie [21] welche die o.g. Klinischen Klassifikationen mit den intraoperativen Befunden bei ECF-Patienten hinsichtlich der Stabilität der Wachstumsfuge verglich, zeigte, dass die Korrelation zwischen klinischen Klassifikationen und intraoperativem Befund ungenau ist. Eine weitere Einteilung der ECF bezieht sich auf den Abrutschgrad des Femurkopfes im Vergleich zum Schenkelhals. Eine Dislokation $<30^{\circ}$ wird als mild, $30-50^{\circ}$ moderat und ein Abrutschen $>50^{\circ}$ als schwere ECF bezeichnet. Leider werden die Röntgenaufnahmen meistens nicht standardisiert durchgeführt, sodass auch hier Messfehler hinsichtlich Ausmaß des Abrutschens entstehen können.

\section{Behandlung}

Studien über den natürlichen Verlauf der Erkrankung ohne Therapie zeigten die Tendenz des Femurkopfes, immer mehr abzurutschen, was zu einer deutlichen klinischen Verschlechterung des Patienten führt [22 -24]. Somit ist das Hauptziel der Behandlung der ECF auch bei minimalen Abrutschgraden, das weitere Abgleiten des Femurkopfes zu verhindern und wenn möglich, bei schweren Dislokationen den mechanischen Konflikt zwischen proximalem Femur und Azetabulum zu beheben. Hierdurch wird gewährleistet, dass die Funktion der Hüfte normalisiert und ein Fortschreiten von Gelenkschäden möglichst verhindert wird. Die 1964 von Dunn publizierten exzellenten Ergebnisse [25,26] zur Wiederherstellung der proximalen femoralen Anatomie mittels offenem chirurgischen Verfahren bei ECF konnten von anderen Autoren nicht reproduziert werden (hohe Inzidenz von avaskulären Nekrosen des Femurkopfes), sodass die Standardbehandlung der ECF gemäß Literatur das „Insitu-Pinnen" des Femurkopfes auf dem Schenkelhals darstellt. Je nach Stabilität und Abrutschgrad wird vorgängig eine geschlossene Reposition vollzogen, um die Position 

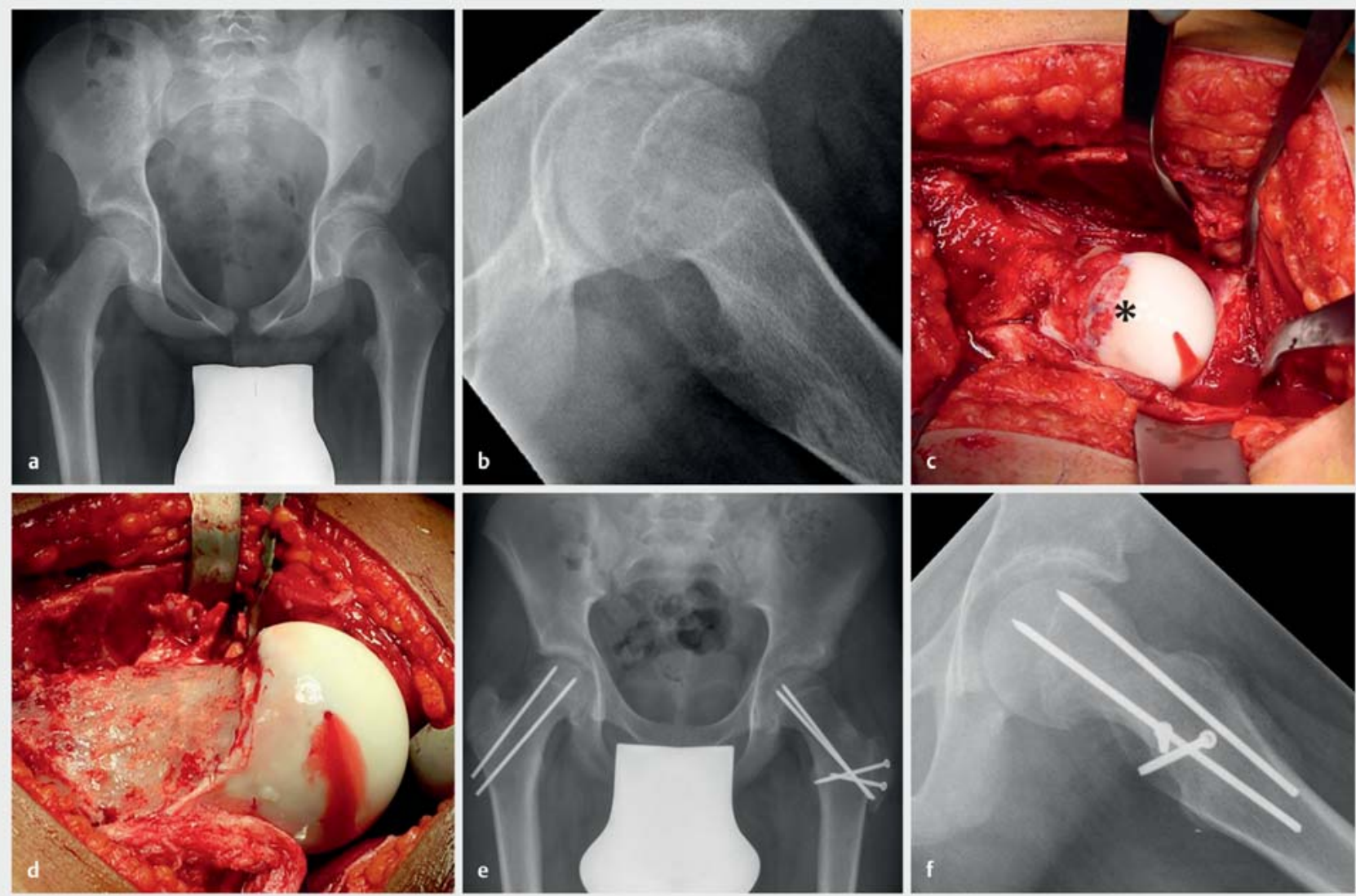

- Abb. 14 Knabe 14 Jahre, ECF links: a, b sichtbare Abkippung des Femurkopfes nach dorsal. Die Wachstumsfuge ist bereits abgeschrägt als Zeichen des chronischen Verlaufes. Ventral zeigt sich eine starke Offset-Störung im Sinne eines CAM-Impingements. c Intraoperatives Bild vor OffsetVerbesserung. Das Periost ( $\left.{ }^{*}\right)$ ist elongiert. Der Femurkopf ist stabil auf dem Schenkelhals verankert. Nach Anbohren blutet der Femurkopf aus dem Bohrloch. d Intraoperatives Bild nach Verbesserung des Kopf-Schenkelhals-Offsets. e, f Postoperatives Röntgenbild a.-p. und Lauenstein links. Verbesserter ventraler Offset nach chirurgischer Hüftluxation links mit Fixation des Femurkopfes wie in $>$ Abb. 1. Milde Trochanterdistalisation und Fixation mit $2 \times 3,5$-mm-Kortikalisschrauben. Persistenz der dorsalen Fehlstellung der Wachstumsfuge (hat keinen Einfluss wegen Fixation des Femurkopfes). Prophylaktisches Pinnen auf der Gegenseite.

des Femurkopfes zu verbessern. Der Nachteil dieses Verfahrens ist, dass die Fehlstellung des Femurkopfes nicht vollständig korrigiert wird. Somit ist der Patient häufig postoperativ weiterhin durch ein aussenrotiertes Gangbild beeinträchtigt. Auch können die noch jungen Patienten durch die persistierende Fehlstellung Probleme im Alltag (Sitzen oder Fahrradfahren) oder bei sportlichen Aktivitäten bekommen. Viele Patienten müssen sich aus diesem Grund Folgeoperationen unterziehen. Um nicht die Femurkopfdurchblutung durch den Folgeeingriff zu kompromittieren, werden dann häufig inter-/subtrochantäre Osteotomien durchgeführt, die eine sekundäre Pathologie kreieren, da sie nicht am eigentlichen Ort der Fehlstellung, dem Schenkelhals, korrigieren. In den letzten 18 Jahren konnte in vielen Publikationen gezeigt werden, dass bereits ein beträchtlicher intraartikulärer Schaden bei einer ECF vorliegen kann [27 - 29] und dass dieser bei Persistenz der Fehlstellung des Femurkopfes nach Insitu-Fixation, vor allem bei starken Fehlstellungen, im weiteren Verlauf noch zunehmen kann. Die Folge ist eine frühzeitige degenerative Veränderung des Hüftgelenkes [30]. Im Zuge der Entwicklung der chirurgischen Hüftluxation durch Ganz [31] wurde eine Operationstechnik basierend auf den Studien von Dunn entwickelt, um den Femurkopf bei starkem Abrutsch anatomisch auf den Oberschenkelhals zu reponieren [32,33]. Zusätzlich können in diesem offenen Verfahren die intraartikulären Schäden femoral und azetabulär beurteilt und ggf. behandelt werden (z.B. Labrumrefixation). Die klinischen und radiologischen Ergebnisse dieser modifizierten Dunn-Operation sind vielversprechend [34-36]. Die Operationstechnik ist anspruchsvoll, zeigt aber bei korrekt ausgeführter Technik vergleichbare oder sogar niedrigere Komplikationsraten als das In-situ-Fixieren. Es sei aber darauf hingewiesen, dass der Chirurg zur komplikationsfreien Durchführung dieser Operation über eine große operative Erfahrung in der hüftgelenkserhaltenden Chirurgie verfügen muss. Aus diesen Gründen sollte die 

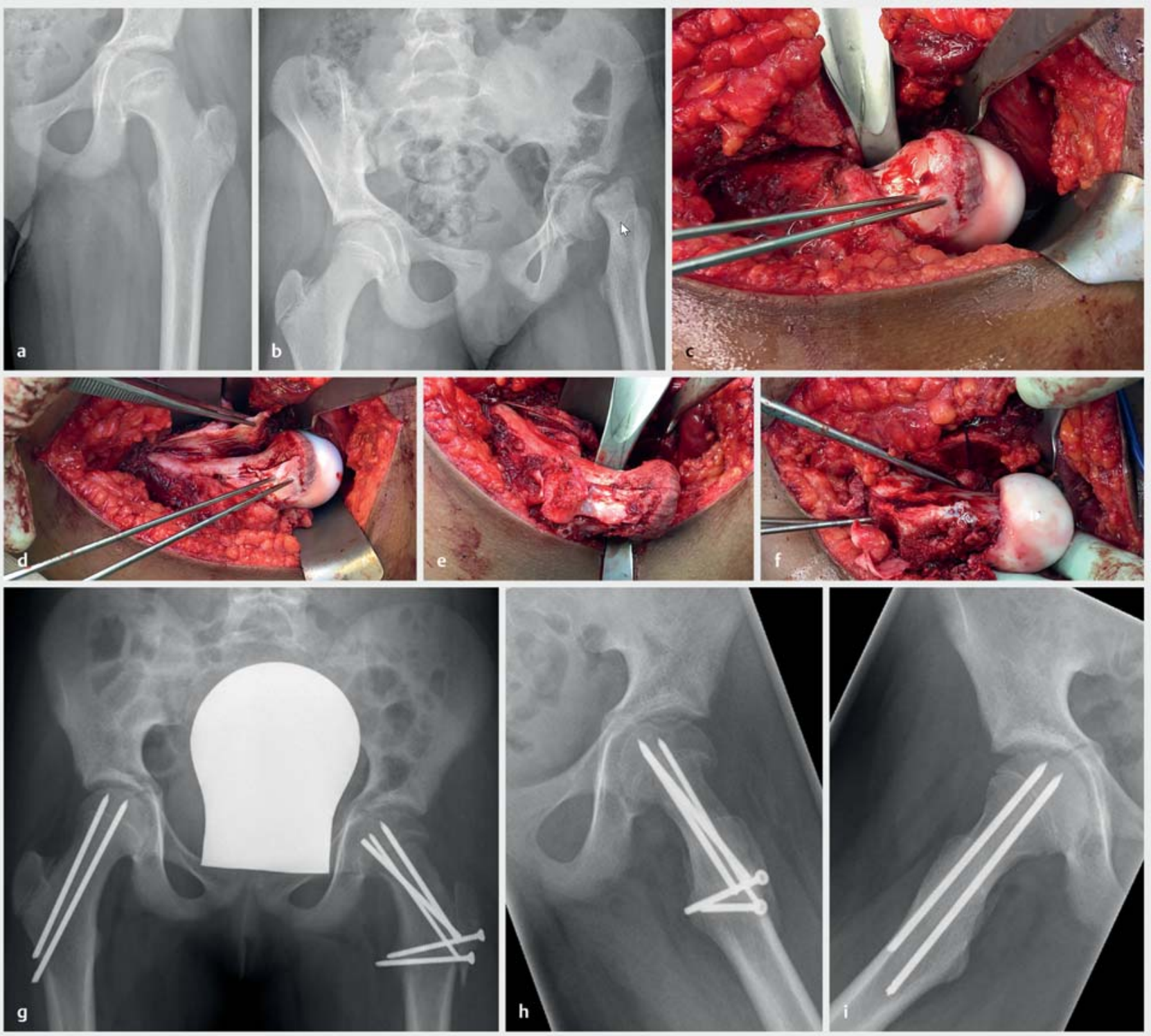

- Abb. 15 Mädchen 11 Jahre. Seit Monaten Hüftschmerzen. Beim Treppenhinablaufen einschießende Schmerzen. a A.-p. Röntgenbild linke Hüfte 3 Monate vor dem akuten Ereignis. Die Auflockerung der Fuge und das milde Abrutschen des Femurkopfes wurde nicht wahrgenommen. Eine zweite Ebene wurde aus strahlenschutzgründen nicht durchgeführt. b Akutes Abrutschen des Femurkopfes links auf der a.-p. Aufnahme. Eine zweite Ebene wurde aus Schmerzgründen nicht durchgeführt. c Intraoperatives Bild: Das Periost $\left({ }^{*}\right)$ ist zerrissen und der Femurkopf instabil. Vor Luxation aus dem Azetabulum muss der Femurkopf unter Sicht prophylaktisch mit 2 kleineren Kirschner-Drähten gepinnt werden, damit er nicht vollständig disloziert und die femurkopfversorgenden Gefäße abreißen. d Intraoperatives Bild: Ventral wurde bereits der retinakuläre Flap (Periost) abgelöst (+). Der Femurkopf blutet aus dem Bohrloch. e Intraoperatives Bild: Femurkopf vollständig vom Oberschenkelhals abgelöst und ins Azetabulum geschoben (nicht sichtbar). Die Schenkelhalsmetaphyse (") ist bereits deutlich nach dorsal abgeschrägt (Anzeichen für Chronizität). $\mathrm{f}$ Intraoperatives Bild: Nach Reposition des Femurkopfes anatomische Stellung und Fixation mit Kirschner-Drähten. g-i Postoperative Kontrolle a.-p. und Lauenstein. Korrekte Stellung. Prophylaktische Pinnung auf der Gegenseite.

modifizierte Dunn-Operation nur in ausgewählten Zentren angewendet und Patienten rechtzeitig zur adäquaten Therapie dorthin überwiesen werden. Heutzutage sollte die Therapie der ECF dem Abrutschgrad des Hüftkopfes, sowie der bereits bestehenden Veränderung des Schenkelhals-/Kopfüberganges angepasst werden. Das Behandlungschema der ECF des Inselspitals Bern ist in - Tab. 2 dargestellt. Auch bei einseitiger Erkrankung empfehlen wir in gleicher Narkose die prophylaktische In-situ-Fixation der Gegenseite aus den o. g. Gründen (Risiko der bilateralen Erkrankung).

\section{Komplikationen}

Die Komplikationsraten bei Behandlung der ECF variieren in der Literatur sehr stark. Dies nicht zuletzt, da wie oben erwähnt, die klinischen Klassifikationen nicht eindeutig in 
- Tab. 2 ECF-Behandlungsschema in der Kinderklinik Bern. Risiko der bilateralen Erkrankung.

\begin{tabular}{|l|l|l|}
\hline Gruppe & Charakteristika & Therapie \\
\hline 1 & ECF undisloziert (Fuge lediglich aufgelockert) $\triangleright$ Abb. 13 & In-situ-Fixation mit 2 Vollgewinde-Kirschner-Drähten \\
\hline 2 & ECF-Abrutschung $<20^{\circ}$ (Fuge horizontal, metaphysärer Sporn) & In-situ-Fixation + Hüftarthroskopie zur Offset-Korrektur \\
\hline 3 & $\begin{array}{l}\text { ECF }>20^{\circ} \text { (Fuge bereits abgeschrägt durch chronische Veränderun- } \\
\text { gen) }>\text { Abb. } \mathbf{1 4}\end{array}$ & $\begin{array}{l}\text { chirurgische Hüftluxation mit Offset-Korrektur, bei instabiler } \\
\text { Fuge und/oder großem Ausmaß der mechanischen Beein- } \\
\text { trächtigung: modifizierte Dunn-Operation }\end{array}$ \\
\hline 4 & $\begin{array}{l}\text { ECF }>30^{\circ} \text { (instabile Fuge, große mechanische Beeinträchtigung bei } \\
\text { stabiler Fuge) }>\text { Abb. } \mathbf{1 5}\end{array}$ & modifizierte Dunn-Operation \\
\hline
\end{tabular}

der Lage sind, die komplikationsträchtigen instabilen ECF zu detektieren. Die gefürchtetste Komplikation bei der ECF-Behandlung stellt die avaskuläre Nekrose (AVN) dar. Diese kann bereits durch die Erkrankung selbst resultieren oder auch iatrogen bei der chirurgischen Versorgung hervorgerufen werden. Bei der rein traumatischen ECF wird in der Literatur eine Inzidenz der AVN von $80-100 \%$ angegeben. Liegt die AVN-Rate bei der stabilen ECF bei 0-4,6\% [37] nach In-situ-Fixation, so wird die Inzidenz der AVN bei instabilen ECF mit 4,7-58\% [38] angegeben. In einer kürzlich veröffentlichten eigenen Studie über die Langzeitergebnisse der modifizierten Dunn-Operation zeigten sich bei 42 Patienten nach 10 Jahren Follow-up keine Fälle von avaskulären Nekrosen [39]. Im eigenen Patientenkollektiv (noch nicht publiziert) mit intraoperativ instabilen Wachstumsfugen kam es bei 3/51 Hüften $(5,8 \%)$ zu einer avaskulären Nekrose. Dies zeigt, dass bei technisch korrekter Durchführung der Operation mit intraoperativ aktivem Erhalt der Femurkopfdurchblutung die Komplikationsrate für schwerwiegende Komplikationen wie AVN auch bei den komplikationsträchtigen instabilen ECF sehr niedrig gehalten werden kann. Die Entfernung des Osteosynthesematerials darf nicht vor Verschluss der Wachstumsfugen am proximalen Femur erfolgen, um ein erneutes Abrutschen des Femurkopfes zu vermeiden.

\section{Fazit}

Verletzungen am proximalen Femur und Beckenavulsionsverletzungen bei Kindern sowie die Epiphysiolysis capitis femoris erfordern eine zeitnahe suffiziente Therapie, um Komplikationen wie Fehlstellungen, Pseudarthrosen, Impingementsyndrome und Femurkopfnekrosen zu minimieren. Die Therapieoptionen hängen vom Schweregrad der Verletzung/Erkrankung ab und reichen von konservativ, über Osteosynthesen, bis zur chirurgischen Hüftluxationen oder modifizierten Dunn-Operation.

\section{Interessenkonflikt}

Die Autoren erklären, dass kein Interessenkonflikt vorliegt.

\section{Autorinnen/Autoren}

Kai Ziebarth
$\begin{aligned} & \text { FMH Orthopädische Chirurgie und Traumato- } \\ & \text { logie des Bewegungsapparates, Leiter Kinder- } \\ & \text { orthopädie/Traumatologie der Kinderchirurgi- } \\ & \text { sche Universitätsklinik des Inselspital Bern }\end{aligned}$
$\begin{aligned} & \text { Nadine Kaiser } \\ & \text { Fachärztin für orthopädische Chirurgie und } \\ & \text { Traumatologie des Bewegungsapparates, } \\ & \text { Oberärztin Kinderorthopädie der Kinderchirur- } \\ & \text { gischen Universitätsklinik des Inselspital Bern }\end{aligned}$
$\begin{aligned} & \text { Thoralf Liebs } \\ & \text { Priv.-Doz. Dr. med., Facharzt für Orthopädie } \\ & \text { und Unfallchirurgie, spezielle orthopädische } \\ & \text { Chirurgie, Kinderorthopädie; Oberarzt der } \\ & \text { Kinderchirurgischen Universitätsklinik des } \\ & \text { Inselspital Bern }\end{aligned}$

Korrespondenzadresse

\section{Dr. med. Kai Ziebarth}

Leiter Kinderorthopädie/Traumatologie

Kinderchirurgische Universitätsklinik

Inselspital

Freiburgstrasse

3010 Bern

Schweiz

\section{Literatur}

[1] Beaty JH, Kasser JR, eds., Rockwood and Wilkins' Fractures in Children. Philadelphia: Lippincott Williams \& Wilkins; 2010

[2] Johnson EO, Soultanis K, Soucacos PN. Vascular anatomy and microcirculation of skeletal zones vulnerable to osteonecrosis: vascularization of the femoral head. Orthop Clin North Am 2004; 35: 285-291, viii

[3] Slongo T, Audigé L, Schlickewei W et al., Development and validation of the $\mathrm{AO}$ pediatric comprehensive classification of long bone fractures by the Pediatric Expert Group of the AO Foundation in collaboration with AO Clinical Investigation and Documentation and the International Association for Pediatric Traumatology. J Pediatr Orthop 2006; 26: 43-49 
[4] Arbeitsgemeinschaft für Osteosynthesefragen. Howard A, Hunter J, Slongo T et al. Im Internet: https://www2.aofoundation.org/wps/portal/surgery; Stand: 01.07.2018

[5] Yeranosian M, Horneff JG, Baldwin K et al. Factors affecting the outcome of fractures of the femoral neck in children and adolescents: a systematic review. Bone Joint J 2013: 95-B: 135-142

[6] Dial BL, Lark RK. Pediatric proximal femur fractures. J Orthop 2018; 15: 529-535

[7] Schuett DJ, Bomar JD, Pennock AT. Pelvic apophyseal avulsion fractures: a retrospective review of 228 cases. J Pediatr Orthop 2015; 35: 617-623

[8] McKinney BI, Nelson C, Carrion W. Apophyseal avulsion fractures of the hip and pelvis. Orthopedics 2009; $32: 42$

[9] Metzmaker JN, Pappas AM. Avulsion fractures of the pelvis. Am J Sports Med 1985; 13: 349-358

[10] Gidwani S, Jagiello J, Bircher M. Avulsion fracture of the ischial tuberosity in adolescents-an easily missed diagnosis. BM] 2004; 329: 99-100

[11] Ferlic PW, Sadoghi P, Singer G et al. Treatment for ischial tuberosity avulsion fractures in adolescent athletes. Knee Surg Sports Traumatol Arthrosc 2014; 22: 893-897

[12] Aronsson DD, Loder RT, Breur G] et al. Slipped capital femoral epiphysis: current concepts. J Am Acad Orthop Surg 2006; 14: 666-679

[13] Zupanc O, Kizancic M, Daniel M et al. Shear stress in epiphyseal growth plate is a risk factor for slipped capital femoral epiphysis. J Pediatr Orthop 2008; 28: 444-451

[14] Loder RT. The demographics of slipped capital femoral epiphysis. An international multicenter study. Clin Orthop Relat Res 1996; (322): 8-27

[15] Loder RT, Skopelja EN. The epidemiology and demographics of slipped capital femoral epiphysis. ISRN Orthop 2011; 2011: 486512

[16] Witbreuk MM, van Royen BJ, Van Kemenade FJ et al. Incidence and gender differences of slipped capital femoral epiphysis in the Netherlands from 1998-2010 combined with a review of the literature on the epidemiology of SCFE. J Child Orthop 2013; 7: 99-105

[17] Jerre R, Billing L, Hansson G et al. Bilaterality in slipped capital femoral epiphysis: importance of a reliable radiographic method. J Pediatr Orthop B 1996; 5: 80-84

[18] Jerre R, Billing L, Hansson G et al. The contralateral hip in patients primarily treated for unilateral slipped upper femoral epiphysis. Long-term follow-up of 61 hips. J Bone Joint Surg Br 1994; 76: 563-567

[19] Fahey J], O'Brien ET. Acute slipped capital femoral epiphysis: review of the literature and report of ten cases. J Bone Joint Surg Am 1965; 47: 1105-1127

[20] Loder RT, Richards BS, Shapiro PS et al. Acute slipped capital femoral epiphysis: the importance of physeal stability. J Bone Joint Surg Am 1993 75: 1134-1140

[21] Ziebarth K, Domayer S, Slongo T et al. Clinical stability of slipped capital femoral epiphysis does not correlate with intraoperative stability. Clin Orthop Relat Res 2012; 470: 22742279

[22] Carney BT, Weinstein SL. Natural history of untreated chronic slipped capital femoral epiphysis. Clin Orthop Relat Res 1996; (322): 43-47

[23] Dobbs MB, Weinstein SL. Natural history and long-term outcomes of slipped capital femoral epiphysis. Instr Course Lect 2001; 50: 571-575
[24] Weinstein SL. Natural history and treatment outcomes of childhood hip disorders. Clin Orthop Relat Res 1997; (344): 227-242

[25] Dunn DM. The treatment of adolescent slipping of the upper femoral epiphysis. J Bone Joint Surg Br 1964; 46: 621-629

[26] Dunn DM, Angel JC. Replacement of the femoral head by open operation in severe adolescent slipping of the upper femoral epiphysis. J Bone Joint Surg Br 1978; 60-B: 394-403

[27] Leunig M, Casillas MM, Hamlet M et al. Slipped capital femoral epiphysis: early mechanical damage to the acetabular cartilage by a prominent femoral metaphysis. Acta Orthop Scand 2000; 71: 370-375

[28] Leunig M, Fraitzl CR, Ganz R. [Early damage to the acetabular cartilage in slipped capital femoral epiphysis. Therapeutic consequences]. Orthopade 2002; 31: 894-899

[29] Ziebarth K, Leunig M, Slongo T et al. Slipped capital femoral epiphysis: relevant pathophysiological findings with open surgery. Clin Orthop Relat Res 2013; 471: 2156-2162

[30] Larson AN, Sierra RJ, Yu EM et al. Outcomes of slipped capital femoral epiphysis treated with in situ pinning. J Pediatr Orthop 2012; 32: 125-130

[31] Ganz R, Gill T], Gautier E et al. Surgical dislocation of the adult hip a technique with full access to the femoral head and acetabulum without the risk of avascular necrosis. J Bone Joint Surg Br 2001; 83: 1119-1124

[32] Leunig M, Slongo T, Ganz R. Subcapital realignment in slipped capital femoral epiphysis: surgical hip dislocation and trimming of the stable trochanter to protect the perfusion of the epiphysis. Instr Course Lect 2008; 57: 499-507

[33] Leunig M, Slong T, Kleinschmidt M et al. Subcapital correction osteotomy in slipped capital femoral epiphysis by means of surgical hip dislocation. Oper Orthop Traumatol 2007; 19: 389-410

[34] Slongo T, Kakaty D, Krause F et al. Treatment of slipped capital femoral epiphysis with a modified Dunn procedure. J Bone Joint Surg Am 2010; 92: 2898-2908

[35] Ziebarth K, Zilkens C, Spencer S et al. Capital realignment for moderate and severe SCFE using a modified Dunn procedure. Clin Orthop Relat Res 2009; 467: 704-716

[36] Huber H, Dora C, Ramseier LE et al. Adolescent slipped capital femoral epiphysis treated by a modified Dunn osteotomy with surgical hip dislocation. J Bone Joint Surg Br 2011; 93: 833838

[37] Kennedy JG, Hresko MT, Kasser JR et al. Osteonecrosis of the femoral head associated with slipped capital femoral epiphysis. J Pediatr Orthop 2001; 21: 189-193

[38] Tokmakova KP, Stanton RP, Mason DE. Factors influencing the development of osteonecrosis in patients treated for slipped capital femoral epiphysis. J Bone Joint Surg Am 2003; 85-A: 798-801

[39] Ziebarth K, Milosevic M, Lerch TD et al. High survivorship and little osteoarthritis at 10-year followup in SCFE patients treated with a modified Dunn procedure. Clin Orthop Relat Res 2017; 475: 1212-1228

Bibliografie

DOI https://doi.org/10.1055/a-0624-4213

OP-JOURNAL 2018; 34: 315-327 @ Georg Thieme Verlag KG Stuttgart · New York ISSN 0178-1715 